QUARTERLY OF APPLIED MATHEMATICS

VOLUME LXIII, NUMBER 4

DECEMBER 2005, PAGES 715-720

S $0033-569 X(05) 00982-X$

Article electronically published on September 27, 2005

\title{
NONLINEAR PRICE EVOLUTION
}

\author{
BY \\ G. CAGINALP \\ Mathematics Department, University of Pittsburgh, Pittsburgh, PA 15260-0001
}

\begin{abstract}
The neoclassical price adjustment equation stipulates that prices move toward equilibrium at a rate that is proportional to the excess demand, i.e., the difference between the demand and supply divided by the demand (at that price). However, the demand and supply are generally nonlinear functions of price. We show that the information on this nonlinear variation of demand and supply leads to a more accurate description of price evolution toward equilibrium. With this additional information the optimal forecast for the price of the good or asset is given by a nonlinear equation. This yields an advantage to traders utilizing all of the available information on supply and demand functions, rather than simply the value at the current price.
\end{abstract}

1. Introduction. A basic question in the economics of nonequilibrium prices involves the equations governing the evolution of prices as they move to the equilbrium value. The classical equation is generally written as [1]

$$
\frac{\tau}{P} \frac{d P}{d t}=\frac{D(P)-S(P)}{D(P)}
$$

where $D(P)$ and $S(P)$ are the demand and supply at price $P$, and $\tau$ is a proportionality constant that scales the time variable. The discrete analog of (1.1) is obtained by replacing the left-hand side with $\{P(t+1)-P(t)\} / P(t)$. It simply states that the rate of change of price (as a fraction) is proportional to the excess demand at that price. This equation is our starting point, although one could consider the more general equation involving a nonlinear function of the excess demand with similar results.

A key problem involving this excess demand equation emerges even for linear supply and demand functions (as shown in Figure 1) whose intersection point is denoted by $P_{e q}$. Suppose we vary the supply function, $S(P)$, to a new function, $\widetilde{S}(P)$, which is much less steep (i.e., supply does not vary as much with price) so that $\widetilde{S}(P)$ and $D(P)$ still intersect at the same price and quantity. The fractional change in price, according to

Received February 21, 2005.

2000 Mathematics Subject Classification. Primary 91B24, 37W40; Secondary 91B08, 91B26.

Key words and phrases. Price adjustment, dynamical price equation, nonlinear supply and demand, asset price, optimal forecast, trading price.

E-mail address: caginalp@pitt.edu

(C)2005 Brown University 


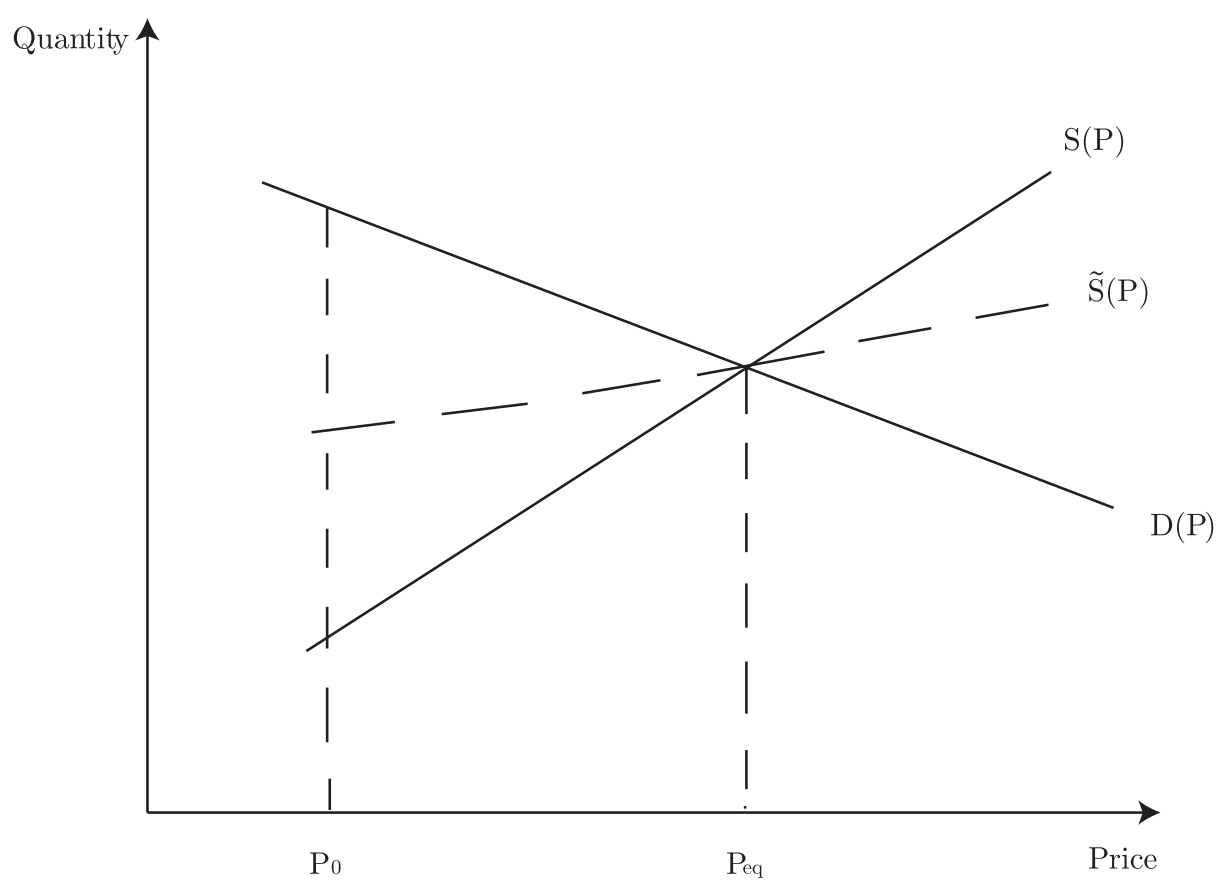

FIG. 1. A key issue of the excess demand price adjustment equation emerges even for linear supply and demand functions, as shown above. The linear supply and demand curves intersect at $P_{\text {eq }}$. Suppose we vary the supply function, $S(P)$, to a new function, $\widetilde{S}(P)$, which is much less steep but still intersects $D(P)$ at the same price and quantity. The fractional change in price, according to the neoclassical equation, is much greater for the original $S(P)$ than for $\widetilde{S}(P)$. Clearly, the adjustment in price cannot be optimal for both situations: either the magnitude of the change is too small in the case of $\widetilde{S}(P)$, or it is too large in the case of $S(P)$.

the (discrete) neoclassical equation (1.1) is much greater for the original $S(P)$ than for $\widetilde{S}(P)$. Clearly, the adjustment in price cannot be optimal for both situations: either the magnitude of the change is too small in the case of $\widetilde{S}(P)$, or it is too large in the case of $S(P)$. Thus large supply and demand relaxation (i.e., steep slopes) tend to exaggerate the predicted price changes within the neoclassical formulation. In general the supply and demand functions are nonlinear, complicating the problem further (as shown in Figure 2).

In many situations, for example in asset markets, one has considerably more information on the supply and demand functions than simply their values at a particular price. In the NASDAQ market for example, one can see the bid-ask array of orders for shares on the Level Two system that could be used to estimate the derivatives of supply and demand. For the linear problem, the concept of deriving a new set of equations is considered in 2 using Newton's method to arrive at a more optimal price. Our linear results recover these equations in a more mathematically smooth way, and generalize naturally to the nonlinear problem. 


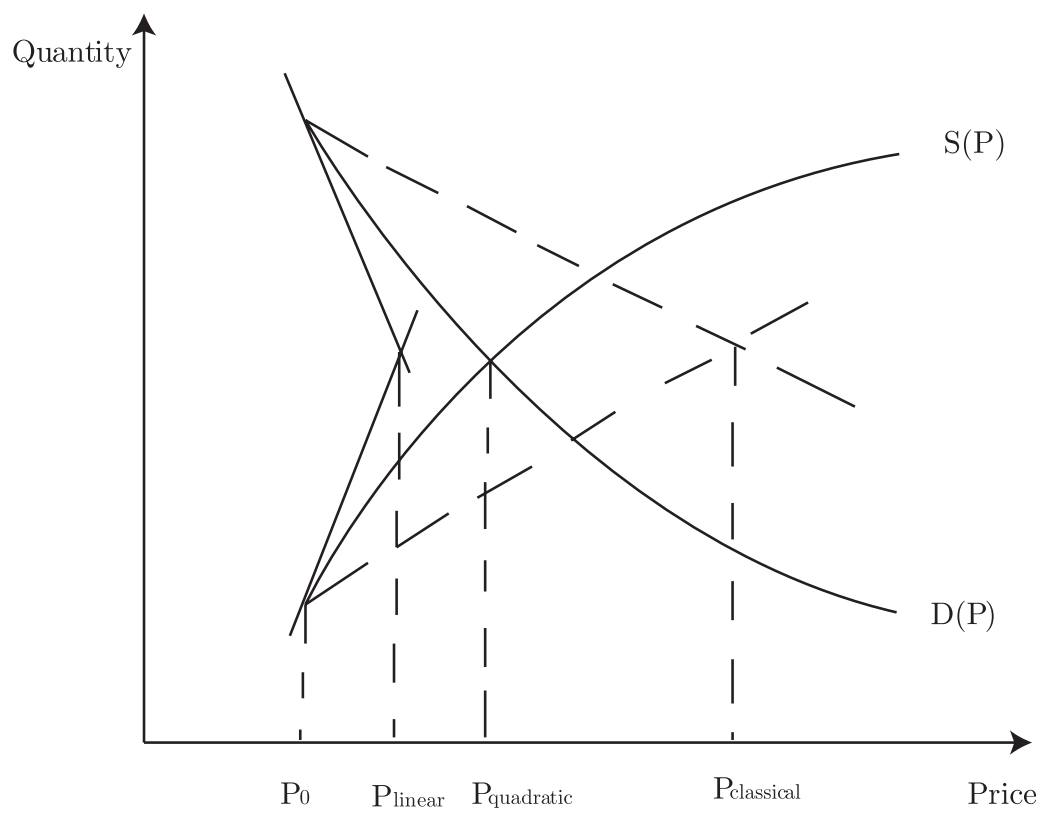

Fig. 2. The relationship between the current price, $P_{0}$, and three calculations for the next period price are illustrated. The traditional price adjustment, $P_{\text {classical }}$ is determined by the excess demand, $(D-S) / D$, at the current price. The linear approximation, $P_{\text {linear }}$, is determined by approximating the supply and demand using only the first derivatives. The quadratic approximation, $P_{\text {quadratic }}$, is obtained from the Taylor expansion up to the second derivatives of supply and demand, thereby taking into account the convexity of these functions. In the case of convex demand and concave supply, as shown in the figure, the quadratic approximation to the price will be higher than the linear. If the supply and demand are exactly quadratic functions, then $P_{\text {quadratic }}$ will be the exact intersection. For arbitrary nonlinear functions, $P_{\text {quadratic }}$ will be the intersection of the quadratic approximation to the supply and demand functions.

2. The linear approximation. We begin with the assumption that traders are aware of supply and demand at $P=P_{0}$ and also have an estimate of their derivatives, $D^{\prime}\left(P_{0}\right)$ and $S^{\prime}\left(P_{0}\right)$, but no information on $D^{\prime \prime}\left(P_{0}\right)$ and $S^{\prime \prime}\left(P_{0}\right)$. Using this information we approximate $D(P)$ and $S(P)$ by straight lines and write

$$
D(P)=D\left(P_{0}\right)+D^{\prime}\left(P_{0}\right)\left(P-P_{0}\right)
$$

and analogously for $S(P)$.

For simplicity we first consider discrete interval times (and set $\tau=1$ ) so the derivatives are replaced by differences. With this information at price $P_{0}$, the participants would move at the next time "period" to the new price, which will be at the point $P_{1}$, where $S\left(P_{1}\right)=D\left(P_{1}\right)$ :

$$
D\left(P_{1}\right)=D\left(P_{0}\right)+D^{\prime}\left(P_{0}\right)\left(P_{1}-P_{0}\right)=S\left(P_{0}\right)+S^{\prime}\left(P_{0}\right)\left(P_{1}-P_{0}\right)=S\left(P_{1}\right) .
$$


Solving for $P_{1}-P_{0}$ we have

$$
P_{1}-P_{0}=-\frac{D\left(P_{0}\right)-S\left(P_{0}\right)}{D^{\prime}\left(P_{0}\right)-S^{\prime}\left(P_{0}\right)} .
$$

Replacing $P_{0}$ with $P(t)$ and $P_{1}$ with $P(t+1)$, we have

$$
P(t+1)-P(t)=-\frac{D(P(t))-S(P(t))}{D^{\prime}(P(t))-S^{\prime}(P(t))} .
$$

Taking the limit to continuous time yields

$$
\tau \frac{d P}{d t}=-\frac{D(P(t))-S(P(t))}{D^{\prime}(P(t))-S^{\prime}(P(t))},
$$

which can also be stated in terms of the fractional change in price upon dividing by $P(t)$. We see that (2.3) differs considerably from (1.1) and provides a more optimal prediction of the price change. This result is identical to that obtained by 2 using Newton's method.

One way of understanding the equations (2.3) and (2.4) is that the demand and supply relaxation are not universal constants. If they were, the original price equation (1.1) would be adequate. The slopes of the supply and demand curves (approximated so far by lines) will vary not only across different assets, but also across different times. Hence, equations (2.3) and (2.4) can be expected to provide a more optimal forecast for the immediate price change if information on the derivatives of supply and demand are available.

3. The quadratic equations. We assume now that for some price $P_{0}$ traders can estimate $D^{\prime \prime}\left(P_{0}\right)$ and $S^{\prime \prime}\left(P_{0}\right)$ in addition to $D\left(P_{0}\right), D^{\prime}\left(P_{0}\right)$, and $S\left(P_{0}\right)$ and $S^{\prime}\left(P_{0}\right)$. Then the most accurate functions describing supply and demand are given by the Taylor series to two terms (i.e., the quadratic approximation):

$$
\begin{gathered}
D(P)=D\left(P_{0}\right)+D^{\prime}\left(P_{0}\right)\left(P-P_{0}\right)+\frac{1}{2} D^{\prime \prime}\left(P_{0}\right)\left(P-P_{0}\right)^{2}, \\
S(P)=S\left(P_{0}\right)+S^{\prime}\left(P_{0}\right)\left(P-P_{0}\right)+\frac{1}{2} S^{\prime \prime}\left(P_{0}\right)\left(P-P_{0}\right)^{2} .
\end{gathered}
$$

We equate (3.1) and (3.2) in order to determine $P_{1}$ when supply and demand are balanced. Denoting $E(P):=D(P)-S(P), E^{\prime}\left(P_{0}\right)=: E^{\prime}$, and $E^{\prime \prime}\left(P_{0}\right)=: E^{\prime \prime}$, etc., we have

$$
E\left(P_{0}\right)+E^{\prime}\left(P_{0}\right)\left(P_{1}-P_{0}\right)+\frac{1}{2} E^{\prime \prime}\left(P_{0}\right)\left(P_{1}-P_{0}\right)^{2}=0 .
$$

Using the quadratic formula for $P_{1}-P_{0}$ which we can write as $P(t+1)-P(t)$, we obtain the equation

$$
P(t+1)-P(t):=P_{1}-P_{0}=\frac{-E^{\prime} \pm\left|E^{\prime}\right| \sqrt{1-\frac{2 E E^{\prime \prime}}{E^{\prime 2}}}}{E^{\prime \prime}} .
$$

The two signs above indicate the two possible intersections of two quadratic functions. One of these will be near the intersection given by the linear equations of Section 2, at least for small $E^{\prime \prime}$, while the other intersection will be irrelevant for the market price. In order to determine the correct sign of the roots, we compare the roots with the 
intersection of the linear functions. First note that one always has $E^{\prime}=D^{\prime}-S^{\prime}<0$, since supply increases with higher price and demand decreases.

Next using a Taylor expansion $\sqrt{1+x}=1+\frac{1}{2} x-\frac{1}{8} x^{3}+\ldots$, one has, for small $E E^{\prime \prime} / E^{\prime 2}$, the second order approximation,

$$
\left(1-\frac{2 E E^{\prime \prime}}{E^{\prime 2}}\right)^{\frac{1}{2}}=1-\frac{E E^{\prime \prime}}{E^{\prime 2}}-\frac{1}{8}\left(\frac{2 E E^{\prime \prime}}{E^{\prime 2}}\right)^{2}+\ldots
$$

Therefore, using $E^{\prime}<0$, one has $\left|E^{\prime}\right|=-E$ and $\pm\left|E^{\prime}\right|=\mp E^{\prime}$, yielding

$$
P(t+1)-P(t) \cong \frac{-E^{\prime} \mp E^{\prime}\left\{1-\frac{E E^{\prime \prime}}{E^{\prime 2}}-\frac{1}{2} \frac{E^{2}{E^{\prime \prime}}^{2}}{E^{\prime 4}}\right\}}{E^{\prime \prime}} .
$$

Since the root with the plus sign is the only one that agrees with the linear approximation, (2.4), we discard the other solution and write

$$
P(t+1)-P(t) \cong-\frac{E}{E^{\prime}}-\frac{1}{2} \frac{E^{2} E^{\prime \prime}}{E^{\prime 3}}
$$

for small $E^{\prime \prime}$ (i.e., $\left|\frac{E E^{\prime \prime}}{E^{\prime 2}}\right| \ll 1$ ). In the continuum form this is simply

$$
\tau \frac{d P}{d t}=-\frac{E}{E^{\prime}}\left(1+\frac{1}{2} \frac{E E^{\prime \prime}}{E^{\prime 2}}\right) .
$$

Hence we can write the full quadratic price equation in the discrete or continuous form as:

$$
\begin{gathered}
P(t+1)-P(t)=-\frac{E^{\prime}}{E^{\prime \prime}}\left(1-\sqrt{1-\frac{2 E E^{\prime \prime}}{E^{\prime 2}}}\right), \\
\tau \frac{d P}{d t}=-\frac{E^{\prime}}{E^{\prime \prime}}\left(1-\sqrt{1-\frac{2 E E^{\prime \prime}}{E^{\prime 2}}}\right) .
\end{gathered}
$$

3.1. Discussion of nonlinear terms. What is the effect of the nonlinearity, i.e., the second term in (3.6)? Since $E^{\prime}$ is always negative and $E^{2}$ positive, the sign of $-\frac{E^{2} E^{\prime \prime}}{2 E^{\prime 3}}$ is that of $E^{\prime \prime}$ and is independent of $E^{2}$. Hence this correction increases the price relative to the linear effect if $E^{\prime \prime}$ is positive (e.g., demand is convex while supply is concave), and vice versa. We summarize these results as follows.

The quadratic correction, namely, the second term in (3.6),

$$
-\frac{E}{E^{\prime}}\left(\frac{1}{2} \frac{E E^{\prime \prime}}{E^{\prime 2}}\right)=-\frac{1}{2} \frac{E^{2} E^{\prime \prime}}{E^{\prime 3}}
$$

adjusts the equilibrium price (relative to the linear equation) upward if $E^{\prime \prime}>0$ and downward if $E^{\prime \prime}<0$. The price change $d P / d t$ or relative price change $P^{-1} d P / d t$ are likewise higher when $E^{\prime \prime}>0$ and lower when $E^{\prime \prime}<0$.

Thus, the effect of the nonlinear term (as shown in Figure 2) is to move the intersection to the right (higher price) if the second derivative of $E$ is positive, e.g., when demand and supply satisfy $D^{\prime \prime}>0$ and $S^{\prime \prime}<0$. 
4. Conclusion. The neoclassical equation for price adjustment stipulates that prices move toward equilibrium at a rate that is proportional to the excess demand, $(D-S) / D$. This equation is inadequate even if one considers it for a single good or asset for two reasons. First, even in the linear case, the slopes of the demand and supply curves need not be constant in time. Second, if these are nonlinear, then additional error is introduced due to the concavity or convexity of supply and demand. The question can be framed in terms of optimal estimates of price evolution. If the only information available is the supply and demand at the particular price, then the neoclassical equation yields the optimal forecast for short term change. However, if there is additional information (or estimate) on the derivatives or second derivatives of these functions, then the use of the neoclassical price adjustment would lead to a suboptimal prediction of price adjustment. (Even if one only has an estimate of concavity or convexity of the excess demand one can still obtain an inequality regarding the predicted price from (3.7).) We prove that the optimal forecast of price change under these conditions is given by equation (3.7) in either the differential or discrete form. In practice this can be used in conjunction with a quadratic regression to forecast changes in price as orders are placed. For example, on the NASDAQ Level Two system, the bid-ask array near the current price reveals not only the imbalance between the supply and demand near that price, but also adequate information to select, using a least squares analysis, quadratic functions for the supply and demand curves. These can then be used in conjunction with (3.7) to determine the rate of change of the price (and of course the direction). In principle, these also forecast the equilibrium price (which is simply the intersection of these quadratic functions). However, in an active market, the orders can change rapidly as the price changes, so that prices may begin to move in a different direction before the equilibrium point is reached. Thus one can repeat this procedure of obtaining the least squares quadratics for supply and demand and utilizing (3.7) for a forecast as often as warranted by the time scale relevant for the particular asset or commodity.

In principle the same ideas can be utilized to carry out this procedure to third or higher order derivatives. Of course, as more derivatives are used, more data is needed to estimate the larger number of parameters. These methods can also be implemented in conjunction with the dependence of supply and demand on price derivatives [3] as well as price.

5. Acknowledgements. I would like to thank Prof. Vernon Smith for bringing this problem to my attention. The support of LGT Capital Mgt. is gratefully acknowledged.

\section{REFERENCES}

[1] Watson, D. and Getz, M., Price Theory and Its Uses, University Press of America, Lanham, MD, (1981).

[2] Plott, C., http://www.hss.caltech.edu/ cplott/, (2004).

[3] Caginalp, G. and Balenovich, D., Asset flow and momentum: deterministic and stochastic equations, Phil. Trans. Proc. Royal Soc. A, 357, 2119-2133 (1999). MR.1712407(2000e:91067) 